\title{
Teleportation of Continuous Quantum Variables
}

\author{
Samuel L. Braunstein \\ SEECS, University of Wales, Bangor LL57 1UT, United Kingdom \\ H. J. Kimble \\ Norman Bridge Laboratory of Physics 12-33, California Institute of Technology, Pasadena, California 91125
}

(Received 8 September 1997)

\begin{abstract}
Quantum teleportation is analyzed for states of dynamical variables with continuous spectra, in contrast to previous work with discrete (spin) variables. The entanglement fidelity of the scheme is computed, including the roles of finite quantum correlation and nonideal detection efficiency. A protocol is presented for teleporting the wave function of a single mode of the electromagnetic field with high fidelity using squeezed-state entanglement and current experimental capability. [S0031-9007(97)05114-4]
\end{abstract}

PACS numbers: 03.67.-a, 03.65.Bz, 42.50.Dv

Quantum mechanics offers certain unique capabilities for the processing of information, whether for computation or communication [1]. A particularly startling discovery by Bennett et al. is the possibility for teleportation of a quantum state, whereby an unknown state of a spin- $\frac{1}{2}$ particle is transported by "Alice" from a sending station to "Bob" at a receiving terminal by conveying 2 bits of classical information [2]. The enabling capability for this remarkable process is what Bell termed the irreducible nonlocal content of quantum mechanics, namely that Alice and Bob share an entangled quantum state and exploit its nonlocal characteristics for the teleportation process. For spin- $\frac{1}{2}$ particles, this entangled state is a pair of spins in a Bell state as in Bohm's version of the Einstein, Podolsky, and Rosen (EPR) paradox [3] and for which Bell formulated his famous inequalities [4].

Beyond the context of dichotomic variables, Vaidman has analyzed teleportation of the wave function of a onedimensional particle in a beautiful variation of the original EPR paradox [5]. In this case, the nonlocal resource shared by Alice and Bob is the EPR state with perfect correlations in both position and momentum. The goal of this Letter is to extend Vaidman's analysis to incorporate finite (nonsingular) degrees of correlation among the relevant particles and to include inefficiencies in the measurement process. The "quality" of the resulting protocol for teleportation is quantified with the first explicit computation of the fidelity of entanglement for a process acting on an infinite dimensional Hilbert space. We further describe a realistic implementation for the quantum teleportation of states of continuous variables, where now the entangled state shared by Alice and Bob is a highly squeezed twomode state of the electromagnetic field, with the quadrature amplitudes of the field playing the roles of position and momentum. Indeed, an experimental demonstration of the original EPR paradox for variables with a continuous spectrum has previously been carried out [6,7], which when combined with our analysis, forms the basis of a realizable experiment to teleport the complete quantum state of a single mode of the electromagnetic field.

Note that up until now, all experimental proposals for teleportation have involved dichotomic variables in $S U(2)$ [2,8-11], with optical schemes accomplishing the Bell-operator measurement with low efficiency. Indeed, the recent report of teleportation via parametric down conversion [12] succeeds only a posteriori with rare post-selected detection events. By contrast, our scheme employs linear elements corresponding to operations in $\mathrm{SU}(1,1)$ [13] for Bell-state detection and thus should operate at near unit absolute efficiency, enabling a priori teleportation as originally envisionaged in Ref. [2].

As shown schematically in Fig. 1, an unknown input state described by the Wigner function $W_{\text {in }}(\alpha)$ is to be teleported to a remote station, with the teleported (output) state denoted by $W_{\text {out }}(\alpha)$. In analogy with the previously proposed scheme for teleportation of the state of a spin- $\frac{1}{2}$ particle, Alice (at the sending station) and Bob (at the receiving terminal) have previously arranged to share an entangled state which is sent along paths 1 and 2 . Within the context of our scheme in $\mathrm{SU}(1,1)$, the entangled state distributed to Alice and Bob is described by the Wigner function $W_{\mathrm{EPR}}\left(\alpha_{1}, \alpha_{2}\right)$ [4]

$$
\begin{aligned}
W_{\mathrm{EPR}}\left(\alpha_{1} ; \alpha_{2}\right) & =\frac{4}{\pi^{2}} \exp \left\{-e^{-2 r}\left[\left(x_{1}-x_{2}\right)^{2}+\left(p_{1}+p_{2}\right)^{2}\right]-e^{+2 r}\left[\left(x_{1}+x_{2}\right)^{2}+\left(p_{1}-p_{2}\right)^{2}\right]\right\} \\
& \rightarrow C \delta\left(x_{1}+x_{2}\right) \delta\left(p_{1}-p_{2}\right),
\end{aligned}
$$

where $\alpha_{j}=x_{j}+i p_{j}$. Here, the real quantities $\left(x_{j}, p_{j}\right)$ correspond to canonically conjugate variables for the relevant pathways and describe, for example, position and momentum for a massive particle, and quadrature amplitudes for the 


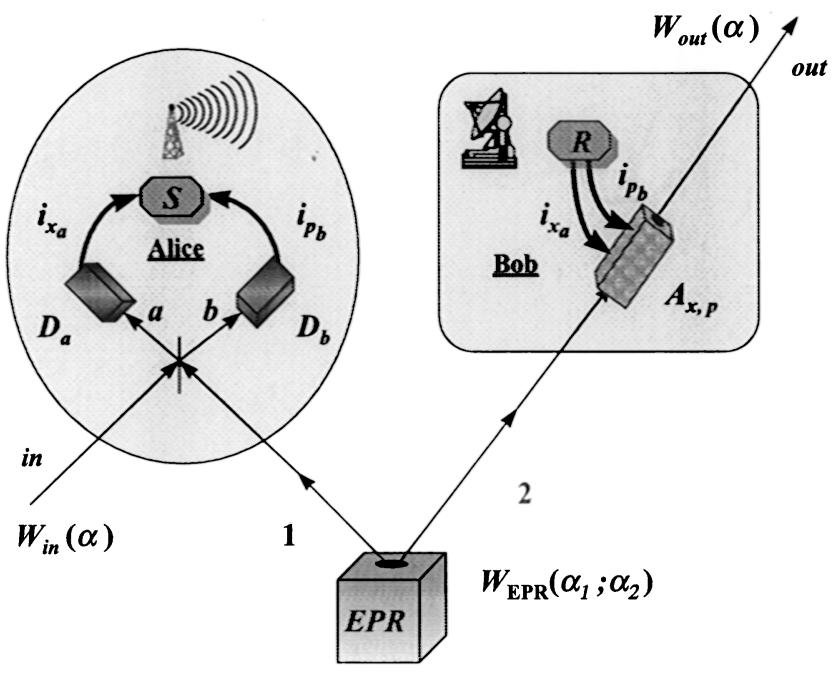

FIG. 1. Scheme for quantum teleportation of an (unknown) input state $W_{\text {in }}(\alpha)$ from Alice's sending station $S$ to Bob's remote receiving terminal $R$, resulting in the teleported output state $W_{\text {out }}(\alpha)$.

electromagnetic field. Note that for $r \rightarrow \infty$, the state described by Eq. (1) becomes precisely the EPR state of Ref. [3] employed by Vaidman [5] and provides an ideal entangled "pair" shared between the teleportation sending and receiving stations, albeit with divergent energy in this limit.

As for the protocol itself, the first step in teleporting the (unknown) state $W_{\text {in }}\left(\alpha_{\text {in }}\right)$ is to form new variables $\beta_{a, b}$ along paths $(a, b)$ which are linear superpositions of those of the initially independent pathways in and 1 at the sending station $S$ of Fig. 1 , namely $\beta_{a, b}=$ $\frac{1}{\sqrt{2}}\left(\alpha_{1} \pm \alpha_{\text {in }}\right)$. The resulting Wigner function in the variables $\left(\beta_{a} ; \beta_{b} ; \alpha_{2}\right)$ exhibits "entanglement" between the paths $(a, b)$ and the remote path 2. Step 2 at $S$ is then to measure the observables corresponding to $\operatorname{Re} \beta_{a}=\frac{1}{\sqrt{2}}\left(x_{1}+x_{\text {in }}\right) \equiv x_{a}$ and $\operatorname{Im} \beta_{b}=\frac{1}{\sqrt{2}}\left(p_{1}-\right.$ $\left.p_{\text {in }}\right) \equiv p_{b}$ at the detectors $\left(D_{a}, D_{b}\right)$ shown in Fig. 1, with the resulting classical outcomes denoted by $\left(i_{x_{z}}, i_{p_{b}}\right)$, respectively. We define ideal measurement of $\left(x_{a}, p_{b}\right)$ to be that for which the distribution $P_{a b}\left(i_{x_{a}} ; i_{p_{b}}\right)$ is identical to the associated Wigner function $W_{a b}\left(x_{a} ; p_{b}\right)$. With the entangled state of paths $(1,2)$ given by Eq. $(1)$, we find

$$
\begin{aligned}
P_{a b}\left(i_{x_{a}} ; i_{p_{b}}\right) & =2 \int d^{2} \alpha W_{\text {in }}(\alpha) G_{\nu}\left[\sqrt{2}\left(i_{x_{a}}-i i_{p_{b}}\right)-\alpha\right] \\
& \equiv 2\left[W_{\text {in }} \circ G_{\nu}\right]\left[\sqrt{2}\left(i_{x_{a}}-i i_{p_{b}}\right)\right],
\end{aligned}
$$

with $\circ$ denoting convolution and $G_{\nu}$ as a complex Gaussian distribution with variance $\nu=\cosh 2 r / 2$. Note that such ideal detectors provide "perfect" information about $\left(x_{a}, p_{b}\right)$ via $\left(i_{x_{a}}, i_{p_{b}}\right)$, while all information about $\left(p_{a}, x_{b}\right) \equiv\left(\operatorname{Im} \beta_{a}=\frac{1}{\sqrt{2}}\left(p_{1}+p_{\text {in }}\right), \operatorname{Re} \beta_{b}=\frac{1}{\sqrt{2}}\left(x_{1}-\right.\right.$ $\left.\left.x_{\text {in }}\right)\right)$ is lost. Furthermore, although $\left(i_{x_{a}}, i_{p_{b}}\right)$ contains a small amount of information about the fiducial state $W_{\text {in }}(\alpha)=W_{\text {in }}\left(x_{\text {in }}, p_{\text {in }}\right)$, this information goes to zero for $r \rightarrow \infty$. Nonetheless, the third and final step at the sending station is to transmit this classical information to the receiving terminal.

As illustrated in Fig. 1, receipt of $\left(i_{x_{a}}, i_{p_{b}}\right)$ allows Bob to construct the teleported state $W_{\text {out }}\left(\alpha_{2}\right)$ from component 2 of the EPR state. That this resurrection is possible can be understood by examining the (unnormalized) Wigner function for the system obtained by integrating out $\left(p_{a}, x_{b}\right)$ in correspondence to Alice's detection of $\left(x_{a}, p_{b}\right)$, namely

$$
G_{\nu}\left(\alpha_{2}\right)\left[W_{\text {in }} \circ G_{\tau}\right]\left(\sqrt{2}\left(i_{x_{a}}-i i_{p_{b}}\right)+\tanh 2 r \alpha_{2}\right),
$$

where the variance $\tau=\operatorname{sech} 2 r / 2$. Note that as $r \rightarrow$ $\infty, G_{\tau}(\alpha)$ quickly approaches a delta function, while $G_{\nu}(\alpha)$ describes a broad background state. Thus, for large $r$, the reduced state of mode 2 is described by a broad pedestal with negligible probability upon which sits a randomly located peak at $\alpha_{2} \approx \sqrt{2}\left(i_{x_{a}}-i i_{p_{b}}\right)$ closely mimicing the incoming state $W_{\text {in }}(\alpha)$. The location of this random "displacement" is distributed according to Eq. (2), and is the classical information that Alice sends to Bob.

By way of the actuator $A_{x, p}$ shown in Fig. 1, Bob thus performs linear displacements of the real and imaginery components of the complex amplitude $\alpha_{2}$ to produce $\alpha_{\text {out }}=\alpha_{2}+\sqrt{2}\left(i_{x_{a}}-i i_{p_{b}}\right)$, where the quantities $\left(i_{x_{a}}, i_{p_{b}}\right)$ are scaled to $\left(x_{a}, p_{b}\right)$. Integrating out $i_{x_{a}}$ and $i_{p_{b}}$ yields the ensemble description of states produced at the output of the teleportation device on an ensemble of input states $W_{\text {in }}$, namely

$$
W_{\text {out }}=W_{\text {in }} \circ G_{\sigma},
$$

where $\sigma=e^{-2 r}$ is the variance of the complex Gaussian $G_{\sigma}$, thus completing the teleportation process.

Clearly, for $r \rightarrow \infty$ the teleported state of Eq. (4) reproduces the original unknown state $W_{\text {in }}$ [5]. However, note that as $r \rightarrow 0, W_{\text {out }}$ also mimics $W_{\text {in }}$, now with two extra units of vacuum noise (i.e., $\sigma=\frac{1}{2}+\frac{1}{2}$ ). One of these noise contributions arises from Alice's attempt to measure both $\left(x_{\text {in }}, p_{\text {in }}\right)$ [14], while the second comes from Bob's use of this necessarily noisy information to generate a coherent state at $\sqrt{2}\left(i_{x_{a}}-i i_{p_{b}}\right)$. In this way quantum mechanics extracts two tariffs (one at each instance of the border crossing between quantum and classical domains), each of which we term the quantum duty or quduty). Note that the limit $r=0$ corresponds to what might be considered "classical" teleportation for which the "best measurement" of the coherent amplitude of the unknown state is made [14] and sent to the receiving station, where it is used to produce a coherent state of that classical amplitude. For any $r>0$, our quantum teleportation protocol beats this classical scheme.

Before calculating an actual figure of merit for our protocol, we now specialize from general continuous variables to the case of a single mode of the electromagnetic field and thereby to actual physical implementations of the various transformations shown in Fig. 1. Beginning 
with the EPR state itself, we note that such a state can be generated by nondegenerate parametric amplification with the quantities $\left(x_{j}, p_{j}\right)$ as the quadrature-phase amplitudes of the field [6], as has been experimentally confirmed via type-II down-conversion [7]. The linear transformation $\beta_{a, b}=\frac{1}{\sqrt{2}}\left(\alpha_{1} \pm \alpha_{\text {in }}\right)$ is accomplished by the simple superposition of modes in and 1 at a 50/50 beam splitter. The detectors $\left(D_{a}, D_{b}\right)$ of Fig. 1 are now just balanced homodyne detectors with the phases of their respective local oscillators set to record $\left(x_{a}, p_{b}\right)$ in the observed photocurrents $\left(i_{x_{a}}, i_{p_{b}}\right)$. Note that for unit efficiency, homodyne detection provides an ideal quantum measurement of the quadrature amplitudes required for our protocol [15-17].

Nonideal detectors, each having (amplitude) efficiency $\eta$, may be modeled by using a pair of auxiliary beam splitters at $\left(D_{a}, D_{b}\right)$ to introduce noise from a pair of vacuum modes described by annihilation operators $\left(\hat{c}_{a, b}, \hat{d}_{a, b}\right)[15,18]$. It is then convenient to introduce annihiliation operators corresponding to the "modes" of the photocurrents described by

$$
\hat{i}_{a, b}=\eta \hat{\beta}_{a, b}+\sqrt{\frac{1-\eta^{2}}{2}}\left(\hat{c}_{a, b}+\hat{d}_{a, b}\right),
$$

where these fictitious objects allow us to apply an analog of the Wigner-function formalism to the photocurrents and to incorporate the effects of nonideal photodetection in a straightforward fashion. For example, loss in the response of Alice's detectors [Eq. (2)] leads to the convolution

$$
\bar{P}_{a b}\left(i_{x_{a}}, i_{p_{b}}\right)=\frac{1}{\eta^{2}}\left[P_{a b} \circ G_{\zeta}\right]\left[\left(i_{x_{a}}+i i_{p_{b}}\right) / \eta\right],
$$

where $G_{\zeta}$ has variance $\zeta=\left(1-\eta^{2}\right) / 2 \eta^{2}$, which goes to zero for $\eta \rightarrow 1$ in correspondence with the ideal character of homodyne detection. Substituting for $P_{a b}$ from Eq. (2) then gives

$$
\bar{P}_{a b}\left(i_{x_{a}}, i_{p_{b}}\right)=\frac{2}{\eta^{2}}\left[W_{\text {in }} \circ G_{\bar{\nu}}\right]\left[\frac{\sqrt{2}}{\eta}\left(i_{x_{a}}-i i_{p_{b}}\right)\right],
$$

where $\bar{\nu}=\frac{1}{2} \cosh 2 r+\left(1-\eta^{2}\right) / \eta^{2}$.

Within the context of the electromagnetic field, Bob can efficiently perform the required phase-space displacement of mode 2 based upon the classical information $\left(i_{x_{a}}, i_{p_{b}}\right)$ received from Alice by combining the field of mode 2 with a (classical) coherent state of mean amplitude $E / t$, where $E=\sqrt{2}\left(i_{x_{a}}-i i_{p_{b}}\right) / \eta$, at a highly reflecting mirror of transmissivity $t \rightarrow 0$. The mean state after this shift is the final teleported state, namely

$$
W_{\text {out }}=W_{\text {in }} \circ G_{\bar{\sigma}}
$$

where $G_{\bar{\sigma}}(\alpha)=\frac{1}{\pi \bar{\sigma}} \exp \left(\frac{-|\alpha|^{2}}{\bar{\sigma}}\right)$ with $\bar{\sigma}=e^{-2 r}+\frac{1-\eta^{2}}{\eta^{2}}$.

The teleportation evolution described by Eq. (8) may be written in density matrix form as

$$
\hat{\rho}_{\text {out }}=\int d^{2} \xi G_{\bar{\sigma}}(\xi) \hat{D}(i \xi) \hat{\rho}_{\text {in }} \hat{D}^{\dagger}(i \xi),
$$

where $\hat{\rho}_{\text {in }}$ is the original state being teleported and $\hat{D}(\alpha)$ is the displacement operator. The dynamics associated with Eq. (9) were first studied by Glauber [19] and Lachs [20] for an "incoming" vacuum state $\hat{\rho}=|0\rangle\langle 0|$ and for squeezed vacuum by Vourdas and Weiner [21]. The detailed behavior of the photocount statistics under this dynamics was investigated by Musslimani et al. [22]. These references also relate the development of the convolutional formalism used here (see also Refs. [23,24]).

To illustrate the protocol, consider teleportation of the coherent superposition state

$$
|\psi\rangle \propto|+\alpha\rangle+e^{i \phi}|-\alpha\rangle,
$$

with corresponding Wigner function $W_{\text {in }}(\alpha)$ illustrated in Fig. 2(a). The teleported Wigner function $W_{\text {out }}(\alpha)$ as computed from Eq. (8) is shown for Fig. 2(b) for parameters corresponding to $-10 \mathrm{~dB}$ of squeezing (i.e., $r=$ 1.15) with efficiency $\eta^{2}=0.99$, which should be compared to the parameters of Ref. [25] [namely squeezing $r=0.69$ (i.e., $6 \mathrm{~dB}$ of squeezing), and detectors with absolute quantum efficiency $\left.\eta^{2}=0.99 \pm 0.02\right]$. Note that the quantum character of the state survives teleportation, including negative values for $W_{\text {out }}$ associated with quantum interference for the off-diagonal components of $\hat{\rho}_{\text {in }}$. For comparison, note that for classical teleportation (i.e., $r=0), W_{\text {out }}^{\text {cl }}$ consists of the (incoherent) superposition of two distributions centered at $\pm \alpha$, each of which is broadened by the quduty.

To provide a quantitative measure of the "quality" of the output state, we note that the strongest measure of fidelity of a teleported state relative to the input state is given by the entanglement fidelity [26]. For processes described by Eq. (9), it is given by

$$
F_{e}=\int d^{2} \xi G_{\bar{\sigma}}(\xi)\left|\chi_{W_{\text {in }}}(\xi)\right|^{2},
$$
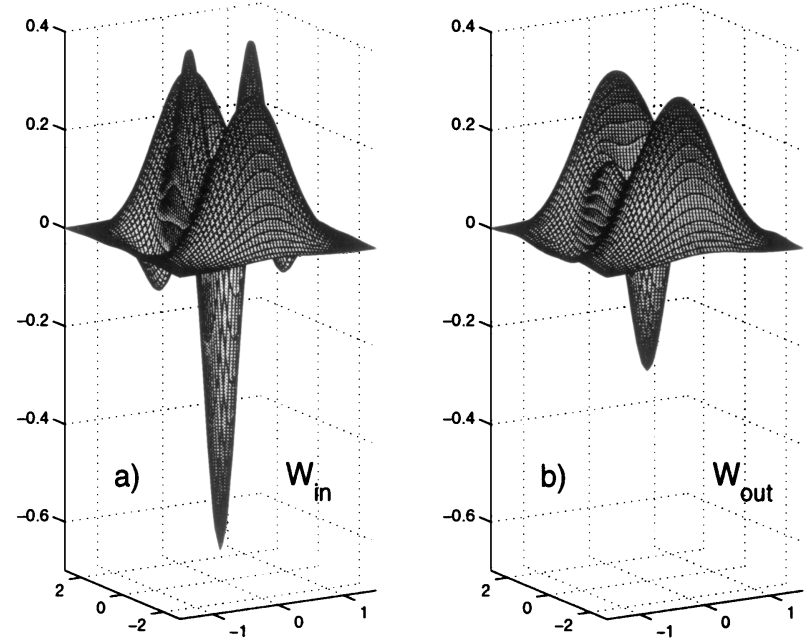

FIG. 2. (a) Wigner function $W_{\text {in }}(\alpha)$ for the input state of Eq. (10) with $\alpha=1.5 i$ and $\phi=\pi$. (b) Teleported output state $W_{\text {out }}(\alpha)$ for $r=1.15$ and $\eta^{2}=0.99$. 
where $\chi_{W_{\text {in }}}(\xi)=\operatorname{tr} \hat{D}(i \xi) \hat{\rho}_{\text {in }}$ is the characteristic function for the incoming state's Wigner function.

For the coherent superposition of Eq. (10) direct substitution yields a fidelity of entanglement $F_{e}$ of

$$
\frac{1}{1+\bar{\sigma}}-\frac{1+e^{-4|\alpha|^{2}}-\exp \left(\frac{-4 \bar{\sigma}|\alpha|^{2}}{1+\bar{\sigma}}\right)-\exp \left(\frac{-4|\alpha|^{2}}{1+\bar{\sigma}}\right)}{2(1+\bar{\sigma})\left(1+e^{-2|\alpha|^{2}} \cos \phi\right)^{2}} \text {. }
$$

For the state shown in Fig. 2(b) this fidelity is 0.6285 for $r=1.15$ and $\eta^{2}=0.99$ compared to 0.2487 for $r=0$ and the same detector efficiency. This latter fidelity precludes observation of any quantum features in the classically teleported state, while the former case yields observable quantum characteristics as seen in Fig. 2.

Beyond any one particular state, let us now concentrate on high fidelity teleportation in general. In this case the Gaussian weighting described by $G_{\bar{\sigma}}$ is sufficiently narrow so that only the lowest terms in an expansion about $\xi=0$ of $\chi_{W_{\text {in }}}$ will contribute. That is, $\left|\chi_{W_{\text {in }}}(\xi)\right|^{2}$ may be approximated by

$$
1-\xi^{* 2}(\Delta \alpha)^{2}-\xi^{2}\left(\Delta \alpha^{*}\right)^{2}-2|\xi|^{2}|\Delta \alpha|^{2},
$$

where $|\Delta \alpha|^{2} \equiv\left\langle|\alpha|^{2}\right\rangle-|\langle\alpha\rangle|^{2}$ averaged over $W_{\text {in }}(\alpha)$. Thus, the condition for high fidelity teleportation (i.e., $\left.1-F_{e} \ll 1\right)$ becomes $1 /|\Delta \alpha|^{2} \gg \bar{\sigma}$. Now $|\Delta \alpha|^{2}$ is just the number of photons (plus $\frac{1}{2}$ ) in the incoming state after it has been shifted so as to have no coherent amplitude. Roughly speaking it is the maximal rms spread of the Wigner function of the unknown quantum state being teleported, and so its reciprocal bounds the size of "important" small scale features in that state, though there can indeed be smaller features. Apparently then the condition for high entanglement fidelity says that features in the Wigner function smaller than $1 /|\Delta \alpha|$ do not give a significant contribution to the state's identity.

In conclusion, our analysis suggests that existing experimental capabilities should suffice to teleport manifestly quantum or nonclassical states of the electromagnetic field with reasonable fidelity. For such experiments, extensions of our analysis to the teleportation of broad bandwidth information must be made and will be discussed elsewhere. In qualitative terms, our scheme should allow efficient teleportation every inverse bandwidth, in sharp contrast to relatively rare transfers for proposals involving weak down conversion for spin degrees of freedom. Although our analysis is the first to obtain explicitly the fidelity of entanglement on an infinite dimensional Hilbert space, an unresolved issue is whether or not our protocol is "optimum," either with respect to this measure or with regard to other criteria in the area of quantum communication (e.g., the ability to teleport optimally an "alphabet" $\{j\}$ of orthogonal states $\left.W_{\text {in }}^{j}\right)$. More generally, the work presented here is part of a larger program to extend classical communication with complex amplitutes into the quantum domain.
S.L. B. was funded in part by EPSRC Grant No. GR/ L91344 and by a Humboldt Fellowship. H. J. K. acknowledges support from DARPA via the QUIC Institute administered by ARO, from the Office of Naval Research, and from the National Science Foundation. Both appreciate the hospitality of the Institute for Theoretical Physics under National Science Foundation Grant No. PHY9407194.

[1] A. Steane, LANL Report No. quant-ph/9708022; A. S. Holevo, LANL Report No. quant-ph/9708046.

[2] C. H. Bennett, G. Brassard, C. Crepeau, R. Jozsa, A. Peres, and W. K. Wootters, Phys. Rev. Lett. 70, 1895 (1993).

[3] A. Einstein, B. Podolsky, and N. Rosen, Phys. Rev. 47, 777 (1935).

[4] J.S. Bell, Speakable and Unspeakable in Quantum Mechanics (Cambridge Univ. Press, Cambridge, England, 1988), p. 196.

[5] L. Vaidman, Phys. Rev. A 49, 1473 (1994).

[6] M. D. Reid and P.D. Drummond, Phys. Rev. Lett. 60, 2731 (1988); M. D. Reid, Phys. Rev. A 40, 913 (1989).

[7] (a) Z. Y. Ou, S. F. Pereira, H. J. Kimble, and K. C. Peng, Phys. Rev. Lett. 68, 3663 (1992); (b) Appl. Phys. B 55, 265 (1992).

[8] L. Davidovich, N. Zagury, M. Brune, J. M. Raimond, and S. Haroche, Phys. Rev. A 50, R895 (1994).

[9] J. I. Cirac and A. S. Parkins, Phys. Rev. A 50, R4441 (1994).

[10] T. Sleator and H. Weinfurter, Ann. N. Y. Acad. Sci. 755, 715 (1995).

[11] S. L. Braunstein and A. Mann, Phys. Rev. A 51, R1727 (1995); 53, 630(E) (1996).

[12] D. Boumeester et al., Nature (London) 390, 575 (1997).

[13] B. Yurke, S. L. McCall, and J. R. Klauder, Phys. Rev. A 33, 4033 (1986).

[14] E. Arthurs and J. L. Kelly, Jr., Bell. Syst. Tech. J. 44, 725 (1965).

[15] H. P. Yuen and J. H. Shapiro, IEEE Trans. Inf. Theory 26, 78 (1980).

[16] S. L. Braunstein, Phys. Rev. A 42, 474 (1990).

[17] Z. Y. Ou and H. J. Kimble, Phys. Rev. A 52, 3126 (1995).

[18] K. Banaszek and K. Wódkiewicz, Phys. Phys. A 55, 3117 (1997).

[19] R. J. Glauber, Phys. Rev. 131, 2766 (1963).

[20] G. Lachs, Phys. Rev. 138, B1012 (1965).

[21] A. Vourdas and R. M. Weiner, Phys. Rev. A 36, 5866 (1987).

[22] Z.H. Musslimani, S. L. Braunstein, A. Mann, and M. Revzen, Phys. Rev. A 51, 4967 (1995).

[23] M. S. Kim and N. Imoto, Phys. Rev. A 52, 2401 (1995).

[24] K. Banaszek and K. Wódkiewicz, Phys. Rev. Lett. 76, 4344 (1996).

[25] E. S. Polzik, J. Carri, and H. J. Kimble, Phys. Rev. Lett. 68, 3020 (1992); (b) Appl. Phys. B 55, 279 (1992).

[26] B. Schumacher, Phys. Rev. A 54, 2614 (1996). 